\title{
Calidad y dificultad de los cuestionarios de un MOOC
}

Quality and difficulty of the Questionnaires of a MOOC

\author{
Carlos Velasco*
}

(iD) http://orcid.org/0000-0002-7871-6936

\section{Pedro Gómez**}

(iD) https://orcid.org/0000-0001-9929-4675

Tipo de Artículo: Reportes de investigación y ensayos inéditos

Doi: 10.17533/udea.unipluri.19.2.06

Cómo citar este artículo:

Velasco, C., y Gómez, P. (2019). Calidad y dificultad de los cuestionarios de un MOOC. Uni-pluriversidad, 19(2), 124-143. https://doi.org/10.17533/udea.unipluri.19.2.06

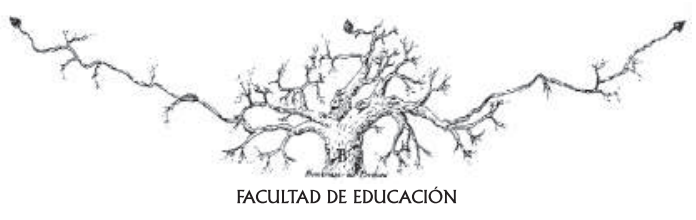

Recibido: 2019-09-23 • Aprobado: 2019-12-14

* Universidad de los Andes. Colombia

E-mail: ca.velasco95@uniandes.edu.co

** Universidad de los Andes. Colombia

E-mail: argeifontes@gmail.com 


\title{
Resumen
}

La oferta de cursos abiertos, gratuitos, masivos en línea (MOOC por sus siglas en inglés) ha tenido un crecimiento importante en los últimos años. La mayoría de estos cursos incluye cuestionarios de evaluación que sirven como guía para establecer un baremo para su aprobación. Estos cuestionarios son una herramienta de aprendizaje porque ponen en juego el conocimiento del participante y le dan la oportunidad para identificar sus dificultades y superarlas. También pueden convertirse en un impedimento para el progreso cuando aquellos participantes que no los aprueban deciden retirarse. En este sentido, resulta relevante tener procedimientos para establecer la calidad y dificultad de los cuestionarios de un MOOC, cuestiones que se abordan en este artículo. Ejemplificamos estas ideas y procedimientos con los datos del primer curso del programa PriMat ${ }^{1}$, Educación Matemática para profesores de primaria. Los resultados ponen de manifiesto la importancia de evaluar la calidad relativa y la dificultad de los cuestionarios de los cursos MOOC con el propósito de contribuir al aprendizaje y la retención de los participantes.

Palabras clave: MOOC, evaluación de un MOOC, calidad de cuestionarios

\begin{abstract}
Supply of massive, online, open courses (MOOCs) has grown significantly in recent years. Most of these courses include evaluation questionnaires that serve as a guide to establish a scale for approval. These questionnaires are a learning tool because they put the knowledge of the participant into play and give him the opportunity to identify his difficulties and overcome them. They can also become an impediment to progress, when those participants who do not approve decide to withdraw. In this sense, it is relevant to have procedures to establish the quality and difficulty of MOOC questionnaires. In this article, we address these two issues. We exemplify these ideas and procedures with the data from the first course of PriMat, the Mathematics Education Program for primary school teachers. The results highlight the importance of assessing the relative quality and difficulty of the MOOC courses questionnaires in order to contribute to the learning and retention of the participants.
\end{abstract}

Keywords: MOOC, Evaluation of a MOOC, Quality of Questionnaires 
En la actualidad, existe un aumento en la oferta y en la demanda de una modalidad de cursos en línea que permite a personas de diferentes lugares del mundo acceder a información de su interés a través de Internet. En la mayoría de los casos, los participantes no necesitan tener experiencia previa con el contenido o el tema que ofrece el curso. La novedad de este tipo de curso en línea, masivo y abierto (MOOC por sus siglas en inglés) es que varias de las mejores universidades del mundo gestionan su contenido y lo ofrecen al público como una opción de formación no formal gratuita $\mathrm{o}$ con un costo que el participante debe asumir por un certificado emitido por la plataforma y avalado por la universidad. En Colombia, la Universidad de los Andes ofrece actualmente (septiembre de 2019) 28 MOOCs en la plataforma Coursera. Coursera es una de las principales plataformas de enseñanza en línea que ofrece la oportunidad de llegar a lugares y personas que no tendrían acceso a cursos de calidad universitaria. Tres de estos MOOCs forman parte del programa PriMat, Educación Matemática para profesores de primaria. Este programa tiene el propósito de ofrecer a profesores de primaria, las herramientas conceptuales y metodológicas para que ellos puedan ofrecer a sus estudiantes mejores oportunidades para que desarrollen sus competencias matemáticas.

Por las características de un MOOC, los profesores que diseñan e implementan el curso no pueden atender de manera personalizada a sus participantes. Esta es la razón por la que un MOOC se compone de diferentes elementos que buscan mejorar la interacción de los participantes con la platafor- ma, para acceder a la información que están buscando y para interactuar con otros participantes al comentar diferentes cuestiones del contenido del curso y de su propio trabajo. Los elementos más comunes son videos en los que el profesor presenta información, encuestas que recogen información sobre la opinión o el conocimiento de los participantes, cuestionarios de evaluación de los conocimientos aprendidos por los participantes, foros, actividades de evaluación por pares, lecturas, bibliografía y recursos adicionales.

Para aprobar un MOOC en Coursera es necesario que el participante obtenga una calificación de al menos $80 \%$ en los cuestionarios de evaluación. En algunos MOOCs, los participantes reciben una retroalimentación de "Correcto" o "Incorrecto" cuando responden las preguntas de un cuestionario. En otros cursos, como los del programa Primat, los participantes reciben una retroalimentación específica a la opción que escogen en cada pregunta del cuestionario. Si el participante no responde correctamente al menos $80 \%$ de las preguntas, debe responderlo de nuevo, tantas veces como sea necesario, para aprobarlo. En el marco de este esquema, los cuestionarios se convierten en una herramienta de aprendizaje. Adicionalmente, los cuestionarios también pueden convertirse en un impedimento para el progreso de un participante en el curso. Si después de varios intentos, el participante no logra aprobar el cuestionario, puede desistir de continuar en el curso y perder la oportunidad de capacitarse con él. Por consiguiente, el diseño de los cuestionarios debe lograr un equilibrio en términos de dificultad. Si son demasiado fáciles, entonces la mayoría de los partici- 
pantes responden todas las preguntas correctamente. En este caso, el cuestionario no ofrece la oportunidad para que participantes que tienen dificultades con el tema objeto del cuestionario puedan reconocerlas y superarlas. Por otro lado, si el cuestionario es demasiado difícil, en el sentido de que una proporción importante de participantes no lo aprueban, entonces algunos de estos participantes pueden desistir de continuar con su formación (Huang, Zhang y Liu, 2017).

Las reflexiones anteriores ponen de manifiesto la importancia de los cuestionarios como elementos de un MOOC. Como mostramos en el siguiente apartado, algunos estudios han encontrado que la dificultad de los cuestionarios puede determinar en qué medida el curso contribuye al aprendiza- je de los participantes (Özgür y Yurdugül, 2015) y les permite terminarlo con éxito (Jordan, 2015; Perna, Ruby, Boruch, Wang, Scull, Ahmad et al., 2014). En este artículo, abordamos esta cuestión al responder a las preguntas ¿cómo establecer la calidad de los cuestionarios de un MOOC a partir de su formulación? y ¿cómo establecer la dificultad de un cuestionario con base en las respuestas de los participantes a sus preguntas?

En los siguientes párrafos presentamos una breve revisión de la literatura, describimos el programa Primat, concretamos las nociones de calidad relativa y dificultad de un cuestionario, formulamos los objetivos del estudio, describimos la metodología que utilizamos y presentamos los resultados. Terminamos con una breve discusión.

\section{REVISIÓN DE LA LITERATURA}

$$
\text { 口 }
$$

En este apartado presentamos una breve revisión de la literatura en la que abordamos la relación entre los cursos MOOC y las siguientes cuestiones: (a) matemáticas, (b) formación de profesores, (c) deserción y (d) cuestionarios y calidad.

\section{Matemáticas}

Los cursos MOOC en matemáticas que se han producido en la última década forman parte de la cuarta fase del desarrollo del uso de la tecnología en Educación Matemática (Borba, Askar, Engelbrecht, Gadanidis, Llinares y Aguilar, 2016). Estos autores indican que estos ambientes requieren nuevas formas de organizar el conocimiento y mencionan un MOOC sobre los estándares estadounidenses Common Core (Boaler, 2014) y un proyecto costarricense en formación de profesores para la implementación de un nuevo currículo (Ruiz, 2013). Por otro lado, el MOOC "Math in motion" busca presentar una manera diferente de interactuar con el conocimiento matemático (Salinas, Quintero y Sanchez, 2015). Una búsqueda en el portal "MOOC List" en noviembre de 2019 con el término "mathematics", produjo una lista de 12 cursos de los cuales solamente dos se referían a matemáticas escolares.

\section{Formación de profesores}

El diseño de cursos MOOC para la formación de profesores debe atender la complejidad del conocimiento y la experiencia del profesor (Fyle, 2013; Hodges, Lowenthal y Grant, 2016). A pesar de que estos MOOCs son bien recibidos por los profesores y se aprecian mejoras en su conocimiento disciplinar y pedagógico (Donitsa-Schmidt y Topaz, 2018; Gonçalves, Chumbo, Torres y Gonçalves, 2016), requieren que los departamentos a cargo se adapten a nuevas formas de evaluar (Tømte, 2019). Muchos 
profesores no conocen (Perez-Parras y Gomez-Galan, 2015) o no desean profundizar en su formación en este tipo de tecnología (Misra, 2018). En algunos casos, la fobia hacia la tecnología y las dificultades en la conectividad pueden afectar su éxito (Wambugu, 2018).

Los MOOCs para formación de profesores de matemáticas están comenzando a aparecer. Este es el caso de los proyectos "Geometria", en Italia, y "Efan Maths", en Francia (Aldon, Arzarello, Panero, Robutti, Taranto y Trgalová, 2017). Dichos proyectos usan la tecnología con el propósito de promover el desarrollo de comunidades de práctica. En el caso de los profesores de primaria, la literatura menciona un proyecto en diseño sobre matemáticas para estos profesores que aparentemente nunca se realizó (Gadanidis, 2014; Gadanidis y Namukas, 2007).

\section{Deserción}

La tasa de deserción en los cursos MOOC tiende a ser muy alta: usualmente entre el 90\% y 95\% de los estudiantes inscritos no terminan un curso. Se constata que alrededor de la mitad de los estudiantes inscritos no inician el curso, que las primeras dos semanas son críticas para el compromiso de los participantes con el curso y que el tipo de evaluación contribuye a predecir la tasa de éxito de los participantes (Jordan, 2015). En particular, los cuestionarios son un hito clave para predecir ese éxito (Perna et al., 2014, p. 6). Al analizar los datos de un MOOC ofrecido por la Universidad de North Carolina en Chapel Hill, Greene, Oswald y Pomerantz (2015) hallaron que la deserción es menor para estudiantes que ya han tenido experiencias con MOOCs, tienen más experiencia educativa y manifiestan un mayor compromiso con su participación. Por otro lado, el éxito de un estudiante parece depender más de un esfuerzo importante en un periodo corto de tiempo, que de un esfuerzo sostenido durante un periodo largo de tiempo (Samuelsen y Khalil, 2020). En un meta-análisis, Khalil y Ebner (2014) encontraron que las principales causas de deserción fueron falta de tiempo y motivación, sensación de aislamiento, falta de interacción, poca preparación y costos escondidos.

Se han desarrollado varios modelos estadísticos con el propósito de predecir, a partir de las características de los estudiantes, su probabilidad de éxito en un MOOC (Moreno-Marcos, Muñoz-Merino, Maldonado-Mahauad, Pérez-Sanagustín, Alario-Hoyos y Delgado Kloos, 2020; Mourdi, Sadgal, El Kabtane y Berrada Fathi, 2019; Wen, Tian, Wen, Zhou, Cai y Liu, 2020; Xie, 2020; Youssef, Mohammed, Hamada y Wafaa, 2019). Por ejemplo, se ha encontrado una correlación positiva entre la motivación del participante y su probabilidad de éxito en el curso (Chaw y Tang, 2019). En este sentido, son pocos los estudios que identifican y caracterizan la retención de un MOOC en términos de sus elementos y características (Hew, Hu, Qiao y Tang, 2020) y proponen posibles soluciones a esta cuestión, como reducir el acceso o el tiempo disponible (Kim, Yang, Bae, Min, Lee y Kim, 2017).

\section{Cuestionarios}

A pesar de que se reconoce que la evaluación es uno de los aspectos más importantes en la calidad de los MOOCs (Yousef, Chatti, Schroeder y Wosnitza, 2014) y en el aprendizaje del participante (Özgür y Yur- 
dugül, 2015), y que los participantes necesitan la evaluación para conocer su rendimiento y compararlo con el de los demás (Perna et al., 2014), su análisis se ha ignorado en buena parte de los estudios sobre la calidad de los MOOCs (Xiao, Qiu y Cheng, 2019). En aquellos estudios que han abordado la evaluación, se reconoce que los esquemas de evaluación son una de sus principales debilidades (Pilli, Admiraal y Salli, 2018): en muchos casos, se usan pruebas que verifican conocimientos básicos (Normandi Atiaja Atiaja y Segundo Guerrero Proenza, 2016) que no son coherentes con la nueva forma de aprender (Wang, Hall y Wang, 2019).

Los cuestionarios de respuesta múltiple es el esquema de evaluación más común en los MOOCs (Yuan, Gao y Chen, 2017, p.
593). Al respecto, el análisis de estos cuestionarios en algunos MOOCs ha mostrado que tienden a discriminar bien a los participantes con pocos conocimientos, pero proporcionan información muy débil para los participantes con más conocimientos (Loken, Oravecz, Tucker y Linder, 2015, p. 26). En algunos casos se constata que los cuestionarios son fáciles porque se tienen en cuenta "los pocos requisitos para participar y la alta tasa de deserción" (Xiao et al., 2019, p. 10). En este sentido, además de medir la magnitud del conocimiento adquirido, los cuestionarios también se pueden utilizar como una forma eficiente de mejorar las bajas tasas de finalización, recopilar datos para mejorar el curso e investigar (Breslow et al., 2013; Jeffrey, Christopher, \& Jeffrey, 2015; Khalil \& Ebner, 2014).

\section{Programa Primat}

Para enseñar matemáticas, el profesor de educación primaria debe tener suficiente conocimiento de los temas objeto de esa enseñanza, con el nivel de reflexión y la amplitud que le permitan comprender y prever el proceso de aprendizaje de sus estudiantes, y diseñar e implementar actividades que promuevan ese proceso. En la actualidad, y tomando como ejemplo a Colombia, la mayoría de los profesores de primaria cursaron programas académicos que no contaban con un currículo con énfasis en matemáticas. Aunque esta asignatura forma parte de algunos planes de estudio, la intensidad horaria o los créditos programados en esos planes no parecen ser suficientes para que los profesores en ejercicio cuenten con las herramientas necesarias para diseñar, implementar y evaluar estrategias de enseñanza acordes con el nivel educativo en el que se desempeñan. Adicionalmente, los altos costos que pue- den generar los programas académicos presenciales, junto con las dificultades para los traslados y el tiempo del que dispone, impiden que un profesor pueda tener acceso a programas de formación formal de carácter presencial.

Las reflexiones anteriores nos llevaron a concebir un espacio de formación permanente y masiva para profesores de primaria en matemáticas de bajo o ningún costo $\mathrm{y}$ de fácil acceso que pudiera contribuir a la mejora del aprendizaje de los estudiantes. Como parte del programa Educación Matemática para profesores de primaria, estamos poniendo a disposición de los profesores un programa de formación que satisface los requerimientos anteriores y está compuesto por tres MOOCs ${ }^{2}$ publicados en Coursera. A continuación, describimos los tres aspectos de las matemáticas escolares que los parti- 
cipantes en el programa tendrán la oportunidad de conocer y desarrollar.

En cada curso se profundiza en algunos de los temas de las matemáticas escolares de primaria. La experiencia con estos temas concretos permitirá a los participantes profundizar por su cuenta en los demás temas objeto de su práctica docente. En el primer curso se abordan los aspectos conceptuales de los temas de las matemáticas escolares, con el propósito de identificar los diferentes significados que tiene un concepto. En el segundo curso se trabajan los aspectos relacionados con el aprendizaje de las matemáticas, con el propósito de que el profesor reflexione sobre los procesos que los estudiantes deben realizar para lograr la comprensión de los contenidos matemáticos trabajados. En el último curso se abordan los aspectos relacionados con la enseñanza y con el desarrollo de las competencias de los profesores para diseñar e implementar tareas matemáticas escolares en primaria.

Cada curso está conformado por cinco módulos compuestos por lecciones que, a su vez, están conformadas por diferentes elementos que le permiten al participante interactuar con su contenido. Los temas del curso son presentados en videos por un profesor experto en Educación Matemática para primaria. Cada video tiene un resumen que se presenta en forma de mapa conceptual. Los cuestionarios usados para realizar el seguimiento de los avances de los participantes en un curso son de tipo test de selección múltiple. Ejemplos de las preguntas que configuran los cuestionarios son (a) preguntas con respuesta de selección múltiple con respuesta única o múltiple, (b) afirmaciones para indicar si son falsas o verdaderas, y (c) selección de afirmaciones que describen de mejor manera alguna idea presentada en el contenido del curso.
Al igual que los diferentes elementos que componen el curso, los cuestionarios fueron diseñados con el propósito de ofrecer oportunidades de aprendizaje a los participantes. Con base en la experiencia y la literatura (Gómez y Gutiérrez, 2014; Segovia y Rico, 2011), establecimos los conocimientos previos que un posible participante del curso podría tener sobre cada uno de los temas que se desarrollan en el curso. De esta manera, tanto los estímulos como las opciones posibles de respuesta buscan que el participante ponga en juego sus conocimientos. La retroalimentación que se ofrece al participante para cada opción de una pregunta se diseñó con el propósito de contribuir a su aprendizaje del tema tratado en cada lección del curso. Al finalizar cada módulo, un participante aprueba el cuestionario al responder correctamente al menos 8 de 10 preguntas con respuesta única. Para aprobar el curso, un participante debe aprobar los cinco cuestionarios. Nuestro análisis se centra en los resultados de estos cuestionarios en el primer curso del programa.

Coursera pone a disposición de los gestores de un MOOC la información básica sobre la participación de los estudiantes en sus cursos. Esta información se presenta de manera gráfica en la plataforma y corresponde a los datos demográficos, historias o comentarios de los estudiantes, cantidad de estudiantes inscritos, estudiantes activos, y calificación que ellos le dan al curso. Por ejemplo, hasta noviembre de 2019, el primer curso del programa tiene más de 70 mil visitas, 14 mil inscritos, 11 mil estudiantes activos y 2500 participantes que terminaron el curso.

En la tabla 1 presentamos los cinco elementos del curso en los que se presenta mayor deserción, con base en la información proporcionada por la plataforma. 


\begin{tabular}{|c|c|}
\hline Elemento & Porcentaje de deserción \\
\hline Cuestionario 1 & 31,38 \\
\hline Cuestionario 2 & 28,21 \\
\hline Comienzo del curso & 23,19 \\
\hline Cuestionario 3 & 15,26 \\
\hline Cuestionario 4 & 8,69 \\
\hline
\end{tabular}

Observamos que los primeros cuatro cuestionarios se encuentran entre las cinco principales causas de deserción. La otra causa se refiere a aquellos participantes que se inscriben en el curso y no lo comienzan (Jordan, 2015).

Adicionalmente, Coursera permite la descarga de una base de datos compuesta por diferentes archivos con valores separados por comas (CSV por sus siglas en inglés) con información sobre la interacción de los participantes con cada uno de los elementos que componen el curso. Esta información da cuenta de (a) cuántas veces un estudiante ingresa a cada actividad o recurso del curso, (b) qué escribe en los foros, (c) los resultados de su evaluación del aprendizaje con motivo de responder los cuestionarios al final de cada módulo, (d) cuántas veces realizó una prueba, (e) cuántos intentos realizó hasta lograr aprobar, (f) los resultados de cada prueba, (g) sus respuestas a las encuestas tipo quiz, (h) si pasó por unas pruebas requisito y obtuvo los puntajes requeridos, (i) sus respuestas a preguntas abiertas, y (j) los resultados de su evaluación sumativa como requisito para obtener un certificado del curso, entre otros datos.

Acontinuación centramos la atención en el estudio de los cuestionarios de los MOOCs, en especial, en las nociones de calidad relativa y dificultad de un cuestionario.

\section{MARCO CONCEPTUAL}

$\square$

Abordamos el estudio de los cuestionarios desde dos perspectivas: su calidad relativa y su dificultad.

\section{Calidad relativa de un cuestionario}

Basados en una revisión de la literatura, Galofré y Wright (2010) desarrollaron un índice de calidad para evaluar preguntas de opción múltiple que utilizaremos en este estudio. Con este índice, la calidad relativa de una pregunta de opción múltiple se representa con un puntaje. El índice se calcula a partir de diez atributos reconocidos como integrantes de una pregunta de opción múltiple bien construida: "presencia de viñeta, enunciado completo, evitar uso de negaciones, concordancia gramatical entre enunciado y opciones, distractores verosímiles, extensión similar entre las opciones, evitar ninguna y todas las anteriores, opciones or- denadas, opciones homogéneas y aplicación de conocimiento superior" (p. 141).

La presencia de viñeta implica, en nuestro caso, la presencia de un contexto en el que se formula la pregunta. La pregunta tiene un enunciado completo si, al cubrir las opciones, es posible responderla con base en ese enunciado. La pregunta evita el uso de negaciones si no se refiere a lo que no es (p. ej., al usar términos como "excepto" o "falso"). Si la pregunta respeta las reglas gramaticales, entonces tiene concordancia gramatical. Los distractores verosímiles son aquellos en los que cada opción parece verdadera para quienes no conocen la respuesta con certidumbre. Las opciones deben tener una extensión similar entre sí (en algunos casos, se puede pensar que la opción con más detalle es la verdadera). La 
pregunta debe evitar las opciones ninguna y todas las anteriores (estas opciones pueden dar indicaciones sobre la respuesta correcta o ser interpretadas de maneras diferentes). Las opciones deben estar ordenadas numérica o lógicamente. Las opciones deben ser homogéneas, es decir, deben abordar el mismo contenido. Finalmente, la aplicación de conocimiento o superior se refiere a que la pregunta no requiere simplemente el recuerdo de un hecho particular. Los autores describen cómo calcular el índice a partir de un análisis de los defectos presentes en cada pregunta en una escala de 1 a 5 , siendo 5 el puntaje máximo y 1 el mínimo.

\section{Dificultad de preguntas y cuestionarios}

Para abordar la noción de dificultad de una pregunta y un cuestionario, nos basamos en la teoría clásica de pruebas (CTT, por sus siglas en inglés), como paradigma de la psicometría (Franzen, 2011). De acuerdo con esta teoría, para calcular la dificultad de una pregunta con ítems dicotómicos, se cuenta la cantidad de participantes que respondieron correctamente (o en la dirección clave) y se divide por la cantidad de participantes. Esto produce una proporción que se puede expresar como un porcentaje. Por lo tanto, el rango de la dificultad de una pregunta es de $0 \%$ a $100 \%$.

Con base en la teoría clásica de pruebas y para exámenes típicos de un plan de estudios académicos, Thompson (2019) sugiere la siguiente escala para clasificar la dificultad de una pregunta con base en el porcentaje de respuestas correctas: muy fácil (más de 90\%), moderadamente fácil (de 70\% a 90\%), moderadamente difícil (de $50 \%$ a $70 \%$ ), muy difícil (de $30 \%$ a $50 \%$ ) y extremadamente difícil (hasta 30\%). En este artículo, y para cursos MOOC que no tienen requisitos de inscripción y tienden a tener altas tasas de deserción como el nuestro, adaptamos esta propuesta para interpretar la dificultad de una pregunta en los rangos que presentamos en la tabla 2 .

\begin{tabular}{|l|l|}
\hline $\begin{array}{l}\text { Tabla } 2 \text { Rangos de dificultad de una pre- } \\
\text { gunta }\end{array}$ \\
\hline Rango de aprobación & Interpretación \\
\hline $91 \%-100 \%$ & Muy fácil \\
\hline $86 \%-90 \%$ & Moderadamente fácil \\
\hline $76 \%-85 \%$ & Moderadamente difícil \\
\hline $66 \%-75 \%$ & Difícil \\
\hline $0 \%-65 \%$ & Extremadamente difícil \\
\hline
\end{tabular}

La dificultad de un cuestionario depende de la dificultad de las preguntas que lo componen. Vamos a considerar que un cuestionario está bien diseñado si todas sus preguntas se encuentran en los rangos de moderadamente fácil y moderadamente difícil (76\% - 90\%) y tiene una dispersión moderada. Consideramos que, en este rango, los profesores que tienen dificultades importantes tendrán la oportunidad de abordarlas y superarlas y que pocos profesores con resultados en este rango desistirán de continuar aprendiendo en los cursos. Justificamos esta afirmación más adelante.

\section{OBjetivos}

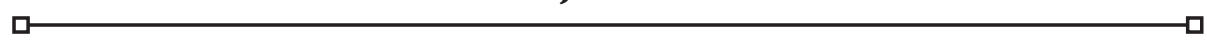

Concretamos el propósito de nuestro análisis en los siguientes objetivos.

1. Establecer la calidad relativa de los cuestionarios usados en Primat
2. Establecer la dificultad de los cuestionarios

3. Establecer relaciones entre la dificultad de los cuestionarios y el índice de calidad relativa de sus preguntas. 


\section{Metodología}

Los datos que dan cuenta de la interacción de un participante con los elementos de un MOOC se registran en una base de datos organizada y compleja que Coursera pone a disposición de los gestores de un curso. Sin embargo, para analizar la información que nos interesa y conocer los resultados de los participantes que responden los cinco cuestionarios en el primer intento, es necesario identificar y aislar la información sobre el primer intento de respuesta de los participantes a los cuestionarios dentro de toda la información disponible. Esta tarea requiere de procedimientos que permitan interactuar con los datos y utilizarlos para realizar diferentes consultas y cálculos. Esa información nos permite identificar las características de los cuestionarios finales, en términos de los promedios de aprobación de los participantes $y$, en ese sentido, determinar su dificultad al contrastar los cuestionarios y revisar la calidad relativa de cada pregunta como un posible factor que incida en su aprobación.

Para determinar la calidad relativa de los cuestionarios, analizamos su diseño: la formulación del estímulo y las opciones de respuesta. Para determinar la dificultad de las preguntas de los cuestionarios, nos basamos en el promedio de aprobación registrado de los participantes. A continuación, describimos estos procedimientos.

\section{Calidad relativa de las preguntas de los cuestionarios}

Las opciones de respuesta de los cuestionarios fueron construidas a partir de nuestra conjetura sobre los conocimientos parciales de los participantes del curso sobre cada uno de los temas de las matemáticas que se tratan en cada módulo. Este tipo de opciones de respuesta de los cuestionarios se conoce como opción plausible. Es decir, las opciones de respuesta tienen sentido y son válidas o creíbles para quien responde la pregunta. Cada cuestionario está formado por un enunciado o estímulo y por varias opciones de respuesta. El siguiente es un ejemplo de una pregunta del segundo cuestionario.

Cuando resolvemos el producto $3 \times 5$ al sumar $5+5+5=15$, ¿de cuál significado del producto hacemos uso?

Opción a. Producto como suma repetida.

Opción b. Producto como resta reiterada.

Utilizamos la propuesta de Galofré y Wright (2010) que presentamos en el marco conceptual, para calcular el índice de calidad relativa de cada una de las 10 preguntas de los cinco cuestionarios finales del primer curso. La pregunta del ejemplo tiene una calificación de 3 puntos en el índice de calidad relativa porque las opciones de respuesta no son verosímiles con respecto al enunciado. Esto quiere decir que la opción b no cumple la condición de parecer una respuesta posible. Esta opción se descarta con facilidad porque hace referencia a una resta reiterada y la pregunta presenta una suma reiterada.

\section{Dificultad de los cuestionarios}

Para establecer la dificultad de los cuestionarios, partimos de la siguiente conjetura. Si un cuestionario está bien diseñado y mide el conocimiento matemático del participante en el tema correspondiente, la distribución del porcentaje de aprobación de los participantes nos puede dar información sobre la complejidad de las preguntas del cuestiona- 
rio, como lo establecimos en el marco conceptual. Por ello, es necesario acceder a los datos de los cuestionarios que proporciona Coursera.

Los archivos de la base de datos disponible en Coursera están acompañados de un manual detallado para acceder a la información de los cursos. Sin embargo, es necesario contar con conocimientos sobre el manejo de bases de datos por medio de consultas en un lenguaje de consulta estructurada (SQL, por sus siglas en inglés) para recuperar la información de los miles de datos registrados y relacionados en los archivos. Para realizar las consultas necesarias para nuestro análisis, usamos un sistema de gestión de bases de datos relacional y un software para gestionar esa base de datos. Una vez identificados los archivos que contienen la información sobre los cuestionarios finales del curso, realizamos las consultas pertinentes en la base de datos para extraer la información correspondiente a los promedios de aprobación de los participantes en el primer intento de responder cada pregunta de cada cuestionario. Adicionalmente, establecimos una conexión entre la base de datos del servidor local y una aplicación $\left(\right.$ Tableau $\left.^{3}\right)$ que nos permitió organizar y visualizar los datos de una manera interactiva. Finalmente, con los datos obtenidos realizamos algunos aná- lisis para comparar los cuestionarios y determinar sus características en términos de la dificultad de las preguntas. Obtuvimos el porcentaje de aprobación de cada pregunta y produjimos gráficos de cajas con estos datos para los cinco cuestionarios.

Debido a las características particulares de los participantes de un curso MOOC, es común que la cantidad de personas que terminan un curso sea mucho menor que la cantidad de personas que se inscriben en el curso (Jordan, 2015). Por esta razón, la cantidad de personas que responden el último cuestionario del curso es menor que las cantidades de personas que responden los cuestionarios anteriores. En la tabla 3, presentamos la cantidad de participantes que habían respondido los cinco cuestionarios a septiembre de 2019.

\begin{tabular}{|c|c|}
\hline \multicolumn{2}{|l|}{ Tabla 3 } \\
Cantidad de participantes que responden \\
\hline Cuestionario & Cantidad de participantes \\
\hline Cuestionario 1 & 4.194 \\
\hline Cuestionario 2 & 3.083 \\
\hline Cuestionario 2 & 2.597 \\
\hline Cuestionario 4 & 2.361 \\
\hline Cuestionario 5 & 2.182 \\
\hline
\end{tabular}

\section{RESUltados}

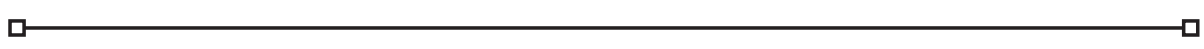

A continuación, presentamos los resultados del análisis de la información para el primer curso del programa Primat. Describimos primero los resultados relacionados con la calidad relativa de los cuestionarios. Después, presentamos los resultados sobre la dificultad de los cuestionarios y los comparamos en términos de esa medida. Finalmente, analizamos la dificultad de cada cuestiona- rio y relacionamos esa dificultad con el índice de calidad relativa de sus preguntas.

\section{Calidad relativa de los cuestionarios}

En la Figura 1 presentamos la distribución del índice de calidad de las 50 preguntas que componen los 10 cuestionarios. 


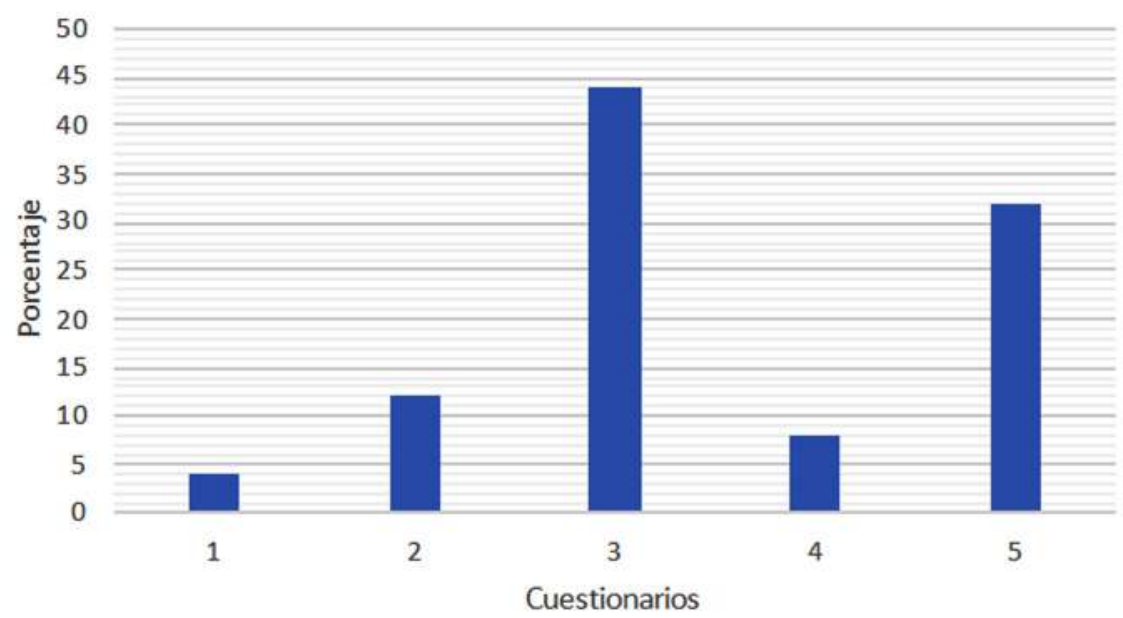

Figura 1. Calidad relativa de las preguntas

Dados los diferentes tipos de preguntas de respuesta única que diseñamos para los cuestionarios y las reglas de asignación de los puntajes del índice que usamos, consideramos que las preguntas tienen un buen nivel de calidad: el $84 \%$ de las preguntas tiene 3 puntos o más y el $40 \%$ obtiene 4 o 5 puntos. No obstante, ocho preguntas (16\%) tienen un índice de calidad inferior a 3. Esta información nos permite identificar las preguntas que, por su diseño, pueden influir en los resultados de aprobación de los cuestionarios.

\section{Dificultad de las preguntas y los cuestionarios}

Las gráficas de cajas de los cuestionarios que presentamos en la Figura 2 nos permiten reconocer la dispersión de los promedios de aprobación de las preguntas de cada cuestionario en el primer intento. Esta información nos induce a formular dos conjeturas en relación con (a) cuál o cuáles son los cuestionarios más difíciles y (b) el grado de dificultad de las preguntas de un cuestionario.

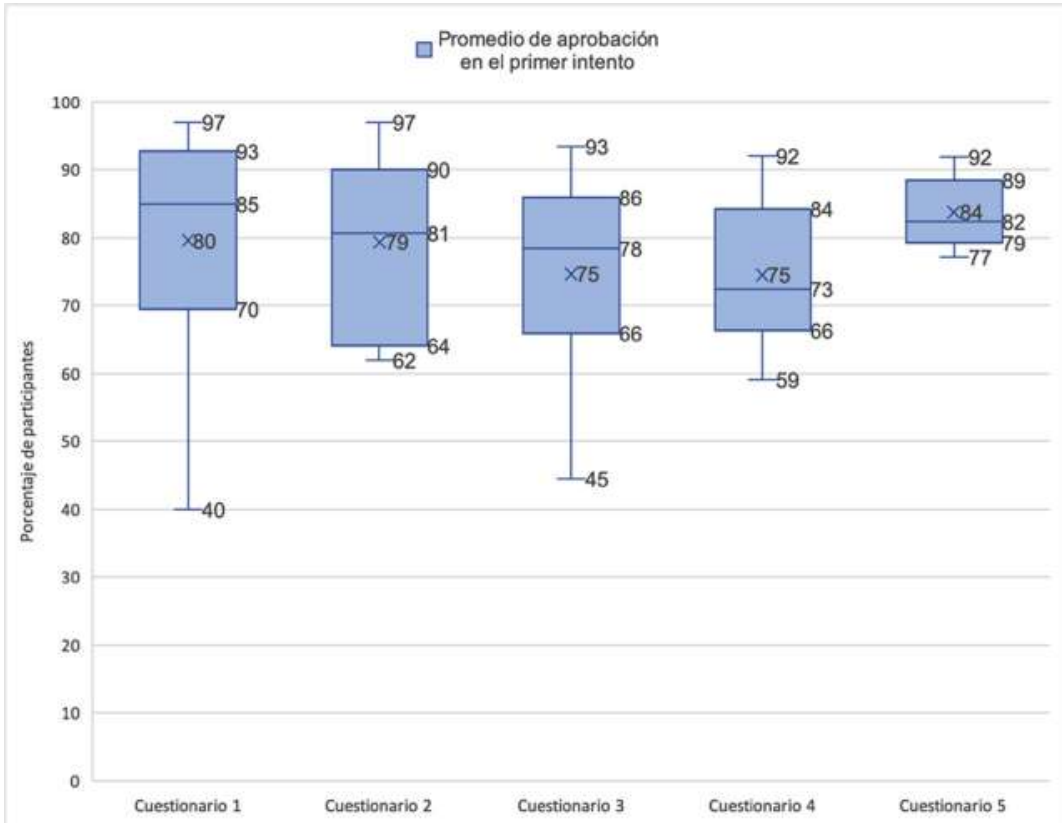

Figura 2. Puntaje promedio del primer intento 
Con base en la información de la figura 2 , a continuación comparamos la dificultad de los cuestionarios (dificultad inter-cuestionarios) y detallamos la dificultad de cada cuestionario (dificultad intra-cuestionarios).

\section{Dificultad inter-cuestionarios}

Como lo sugieren algunos estudios (Chaw y Tang, 2019), conjeturamos que un factor que incide en las diferencias en la dispersión de los promedios de participantes que aprobaron las preguntas de los cuestionarios en el primer intento es producto de su compromiso. Es decir, en la medida en que los participantes avanzan en los cuestionarios, los que deciden mantenerse en el curso son aquellos que han logrado tener éxito en los cuestionarios anteriores. Por ejemplo, hay menos participantes que llegan al cuestionario cuatro que los que hicieron el cuestionario dos (Tabla 3). El cuarto cuestionario tiene agrupados los promedios de aprobación por debajo del valor de aprobación de los demás cuestionarios $\mathrm{y}$, por tanto, consideramos que es el más difícil de los cinco. En la Figura 2, vemos que el $50 \%$ de las preguntas de este cuestionario fueron aprobadas en el primer intento por menos del $73 \%$ de los participantes que respondieron el cuestionario. Estas preguntas se encuentran en las categorías de difícil y extremadamente difícil.

La asimetría negativa de los cuestionarios 1,2 y 3 nos lleva a conjeturar que estos cuestionarios tienen una dificultad relativamente menor, en comparación con los otros cuestionarios. Sin embargo, identificamos algunas preguntas en los cuestionarios $1 \mathrm{y}$ 3 con un promedio de aprobación menor que el promedio de aprobación de los otros cuestionarios y que se ubican en la categoría de extremadamente difícil. En los cuestionarios 1 y 2 , identificamos preguntas con promedios de aprobación mayores que el promedio de los otros cuestionarios y que se ubican en la categoría muy fácil. Analizaremos esas preguntas en el siguiente apartado.

Observamos que los promedios de aprobación de las preguntas del cuestionario 5 tienen la menor variabilidad entre los cuestionarios. Es decir, este cuestionario es el más estable de los cinco. Suponemos que los participantes que respondieron este cuestionario, además de ser menos numerosos que los que respondieron los cuestionarios anteriores, se tomaron más tiempo para seleccionar las respuestas que consideraron correctas, con el propósito de terminar y aprobar el curso sin repetir el cuestionario. La poca dispersión de los valores de aprobación de este cuestionario nos permite constatar que la dificultad de las 10 preguntas está balanceada, porque, aunque tiene una asimetría positiva, no hay promedios de aprobación de preguntas que estén muy alejadas de la mediana, como pasa con los otros cuestionarios. Este cuestionario tiene solamente una pregunta por fuera de los rangos moderadamente fácil y moderadamente difícil.

\section{Dificultad intra-cuestionarios}

Los gráficos de caja también permiten comparar los cuestionarios en términos de la dificultad de sus preguntas. Por ejemplo, identificamos que los cuestionarios 1 y 2 tienen preguntas en la categoría de muy fácil (con promedios de $97 \%$ de participantes que las aprobaron en el primer intento). Por otro lado, los cuestionarios 1 y 3 presentan valores alejados de los promedios de aprobación de las demás preguntas. Estos resultados nos informan de la dificultad que pueden representar algunas preguntas de esos cuestionarios para los participantes.

Para constatar si los altos y bajos promedios de aprobación de algunas pregun- 
tas de los cuestionarios son consecuencia de problemas en el diseño de las preguntas, analizamos las preguntas que registraron valores del $97 \%$ para los cuestionarios 1 y 2 , por debajo del $70 \%$ para el cuestionario $1 \mathrm{y}$ del $66 \%$ para el cuestionario 3 .

Encontramos que, para los cuestionarios 1 y 2 , las preguntas que tiene el porcentaje de $97 \%$ de aprobación tienen una calificación de 3 puntos en el índice de calidad de su construcción. Para el caso del cuestionario 1 , la pregunta no requiere que el participante ponga en juego el conocimiento que desarrolló durante el curso y se pide seleccionar, entre dos opciones, el sinónimo de uno de los términos presentados en una de las lecciones del módulo. Para el caso del cuestionario 2, la pregunta tiene un defecto en su construcción porque las opciones de respuesta no son verosímiles con respecto al enunciado. Esta situación hace que una de las opciones sea descartada con facilidad.

En el cuestionario 1 encontramos dos preguntas con porcentaje de aprobación menor al $70 \%$. Estas preguntas tienen valores del $56 \%$ y $40 \%$ de aprobación y tienen la misma calificación de 3 puntos en índice de calidad porque tienen el mismo defecto en su construcción: la extensión de las opciones no es similar. Sin embargo, los participantes seleccionaron la opción que no es común que se seleccione en estos casos. Esto nos lleva a pensar que, a pesar del error en la construcción de las opciones, la pregunta exige que un participante ponga en juego un conocimiento que se supone desarrolló en la lección y no se espera que responda al hacer uso de memoria.

En el cuestionario 3 encontramos dos preguntas con porcentaje de aprobación menor al $66 \%$ (53\% y $45 \%)$. La primera pregunta tiene 3 puntos en el índice de calidad por un solo error en su construcción relacionado con que la extensión de las opciones no es similar. Al igual que en la descripción del cuestionario anterior, los participantes seleccionaron la opción menos esperada para las opciones que tienen ese tipo de error en su construcción. Esta situación da cuenta del cuestionario y de los participantes en el sentido de que, para los participantes, las opciones presentadas en el cuestionario sí son opciones posibles. Además, esta pregunta también exige que el participante ponga en juego un conocimiento adquirido durante su participación en el curso, en cambio de hacer uso de un recuerdo, como lo señala el índice de calidad que cumple esta pregunta.

La pregunta que tiene un porcentaje del $45 \%$ de aprobación en el cuestionario 3 tiene una calificación de 5 puntos en el índice de calidad. Al revisar la pregunta encontramos que el enunciado hace referencia a uno de los temas de las matemáticas escolares que, en general, presenta mayores dificultades en su aprendizaje: la idea de número racional como decimal (Lortie-Forgues, Tian y Siegler, 2015). Esta es la única pregunta del cuestionario que evalúa ese aspecto de los números racionales.

Además de los promedios altos y bajos que acabamos de analizar, revisamos las características de las preguntas que tuvieron un porcentaje de aprobación en el primer intento de alrededor del 70\%. Encontramos que el $50 \%$ de esas preguntas no tiene defectos de construcción. Esto nos lleva a suponer que la dificultad de las otras preguntas corresponde al tipo de evaluación con el que queremos poner en juego los conocimientos del participante, en el tema estudiado en la lección y el módulo correspondiente. 


\section{Discusión}

No basta con publicar un curso en línea. Los datos de inscripción no son la única información que debe generar tranquilidad a sus gestores. Es necesario prestar atención a la calidad de los elementos que conforman el curso. En el caso de este estudio, centramos el análisis en la calidad relativa y la dificultad de los cuestionarios finales.

El detalle que se alcanzó en los datos nos permite organizar la metodología para abordar el análisis de los cuestionarios finales y de otro tipo de elementos de los otros cursos del programa. Reconocemos que no podemos hacer afirmaciones sobre el nivel de aprendizaje de los participantes del curso, pero creemos que la calidad de los cuestionarios, en términos de su calidad relativa y su dificultad, es un aspecto importante para que los participantes reconozcan los errores en los que incurrieron al responder las preguntas y puedan progresar y aprobar el curso.

Las preguntas que formulamos en los cuestionarios abordan el contenido del curso. No obstante, además de modificar las preguntas que están alejadas del rango de dificultad que determinamos en nuestro análisis, consideramos la posibilidad de incluir información en el curso con el propósito de permitir que los participantes reconozcan de manera más clara el contenido que se aborda.

En la Figura 1 observamos que más del $75 \%$ de las 50 preguntas se ubican entre los niveles 3 y 5 . Al analizar la dificultad de las preguntas encontramos que, de las preguntas con índice de calidad 3, el $31 \%$ son muy fáciles y el $18 \%$ son extremadamente difíciles. Estos dos datos sugieren que para las preguntas estudiadas el índice de calidad relativa no logra discriminar apropiadamente entre las preguntas. Queda pendiente, para trabajos futuros, adaptar el índice de calidad relativa a preguntas de cuestionarios MOOC con las condiciones que hemos mencionado en este artículo.

Los resultados nos llevan a plantear mejoras en los cuestionarios en al menos dos aspectos. Para el caso de las preguntas menos difíciles, debemos modificar los enunciados y opciones con el propósito de permitir que un participante ponga en juego sus conocimientos, en cambio de poder descartar las opciones de las preguntas. Para el caso de las preguntas con un nivel de dificultad mayor, debemos hacer un análisis más detallado de sus enunciados con el propósito de modificarlos para que su nivel de dificultad implique un porcentaje de aprobación entre $76 \%$ y $90 \%$.

En el marco conceptual adaptamos la propuesta de Thompson (2019) sobre la dificultad de una pregunta (y de un cuestionario) a las circunstancias específicas de un MOOC. Estas circunstancias tienen que ver con los dos papeles que un cuestionario juega en un MOOC: como herramienta de aprendizaje y como baremo que determina la deserción de los participantes. Un cuestionario debería ser lo suficientemente difícil como para que aquellos participantes que tienen dificultades importantes las puedan identificar y superar. Esta condición determina el límite superior del rango de dificultad de las preguntas. Por otro lado, el cuestionario debería ser lo suficientemente fácil como para que los participantes continúen su formación en el MOOC después 
de haberlo respondido. Hemos establecido el límite superior del rango en $90 \%$. Esta ha sido una decisión intuitiva basada en la idea de que aquellas preguntas que son respondidas correctamente por más del $90 \%$ de los participantes son demasiado fáciles y no inducen a los participantes a manifestar y superar sus dificultades. Se requiere investigación empírica que sustente esta decisión. Por otro lado, establecimos el límite inferior del rango en $76 \%$. Es posible comprobar la validez de esta decisión si se analiza la relación entre el comportamiento de un participante en un cuestionario (en términos de la proporción de preguntas que respondió correctamente en los diferentes intentos) y su probabilidad de desertar del curso con motivo del cuestionario. Esta es otra cuestión que queda abierta para investigaciones futuras.
Esperamos que la evaluación periódica de las nuevas versiones de los cuestionarios finales del primer curso nos permita identificar mejores resultados en los aspectos analizados en este estudio: el promedio de aprobación de las preguntas del cuestionario en el primer intento.

No hemos encontrado en la literatura estudios que centren su atención en la calidad relativa y la dificultad de los cuestionarios de un curso MOOC, a pesar de que diversos estudios destacan la importancia de la evaluación en la calidad de los cursos (Perna et al., 2014, p. 6; Yousef et al., 2014) y en las tasas de deserción (Breslow et al., 2013). Consideramos que este estudio es una contribución para aquellos gestores de cursos MOOC preocupados por la tasa de deserción y por el papel que los cuestionarios pueden jugar en el aprendizaje de los participantes.

\section{Agradecimientos}

口

Este estudio se realizó con el apoyo del Fondo Francisco José de Caldas (Colciencias), en el marco del programa de investigación 64639, correspondiente a la convocatoria 808 de 2018 para el Proyecto Acometer la inequidad a través de la educación: matemáticas en la educación básica y media 2019-2020. El programa Educación Matemática para profesores de primaria tiene como aliados a Genesis Foundation, el Fondo Puentes de Caña, La Fundación SM y la Fundación Compartir. Agradecemos a María Castro por su colaboración en el manejo de los datos, a Camilo López y Paola Castro por sus ideas sobre cómo presentar los resultados, y a los dos revisores de la primera versión del manuscrito quienes nos proporcionaron comentarios que ayudaron a mejorar el contenido.

\section{Notas}

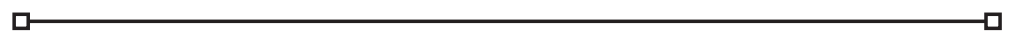

1. PriMat recoge los proyectos de formación e investigación que "una empresa docente" —Centro de formación e investigación de la Facultad de Educación de la Universidad de los Andes-realiza en el área de Educación Matemática en primaria. Los cursos que componen el programa se pueden encontrar en la plataforma Coursera (http://coursera.org) con la búsqueda "matemáticas primaria". 
2. Los tres cursos se identifican en Coursera como Contenido de las matemáticas de primaria, Aprendizaje de las matemáticas de primaria y Enseñanza de las matemáticas de primaria.

3. https://www.tableau.com

\section{REFERENCIAS BIBLIOGRÁFICAS}

Aldon, G., Arzarello, F., Panero, M., Robutti, O., Taranto, E., \& Trgalová, J. (2017). MOOC for mathematics teacher training: design principles and assessment. In G. Aldon \& J. Trgalova (Eds.), The 13th International Conference on Technology in Mathematics Teaching-ICTMT 13 (pp. 1-8). Lyon: ENS.

Boaler, J. (2014). How to learn math: for teachers and parents. Resource document. Stanford University. Downloaded from https://stanford.io/2KtxsNl

Borba, M. C., Askar, P., Engelbrecht, J., Gadanidis, G., Llinares, S., \& Aguilar, M. S. (2016). Blended learning, e-learning and mobile learning in mathematics education. ZDM, 48(5), 589-610.

Chaw, L., \& Tang, C. M. (2019). Driving high inclination to complete massive open online courses (MOOCs): motivation and engagement factors for learners. Electronic Journal of e-Learning, 17, 118-130.

Donitsa-Schmidt, S., \& Topaz, B. (2018). Massive open online courses as a knowledge base for teachers. Journal of Education for Teaching, 44(5), 608-620.

Franzen, M. D. (2011). Item Difficulty. In J. S. Kreutzer, J. DeLuca y B. Caplan (Eds.), Encyclopedia of Clinical Neuropsychology (pp. 100-100). New York, NY: Springer.

Fyle, C. O. (2013). Teacher education MOOCs for developing world contexts: Issues and design considerations. Work presented in Sixth International Conference of MIT's Learning International Networks Consortium (LINC).

Gadanidis, G. (2014). Mathematics-for-Teachers using a MOOC. Society for Information Technology \& Teacher Education International Conference 2014, 2014, 310-314.

Gadanidis, G., \& Namukas, I. (2007). Mathematics-for-Teachers (and Students). Journal of Teaching and Learning, 5(1), 13-22.

Galofré, A., \& Wright, A. C. (2010). Índice de calidad para evaluar preguntas de opción múltiple. Revista de Educación en Ciencias de la Salud, 7(2), 141-145.

Gómez, P., y Gutiérrez, A. (2014). Conocimiento matemático y conocimiento didáctico del futuro profesor español de primaria. resultados del estudio TEDS-M. En M. T. González, M. Codes, D. Arnau y T. Ortega (Eds.), Investigación en Educación Matemática XVIII (pp. 99-114). Salamanca, España: SEIEM. 
Gonçalves, V., Chumbo, I., Torres, E., \& Gonçalves, B. M. F. (2016). Teacher education through MOOC: a case study. In L. Gómez, A. López y I. Candel Torres (Eds.), Proceedings of iCERi2016: 9th International Conference of Education, Research and Innovation (pp. 8350-8358). Sevilla: IATED Academy.

Greene, J. A., Oswald, C. A., \& Pomerantz, J. (2015). Predictors of retention and achievement in a massive open online course. American Educational Research Journal, 52(5), 925-955.

Hew, K. F., Hu, X., Qiao, C., \& Tang, Y. (2020). What predicts student satisfaction with MOOCs: A gradient boosting trees supervised machine learning and sentiment analysis approach. Computers \& Education, 145, 1-15.

Hodges, C., Lowenthal, P., \& Grant, M. (2016). Teacher Professional Development in the Digital Age: Design Considerations for MOOCs for Teachers. Society for Information Technology \& Teacher Education International Conference 2016, 2016(1), 2075-2081.

Huang, L., Zhang, J., \& Liu, Y. (2017). Antecedents of student MOOC revisit intention: Moderation effect of course difficulty. International Journal of Information Management, 37(2), 84-91.

Jordan, K. (2015). Massive open online course completion rates revisited: Assessment, length and attrition. International Review of Research in Open and Distance Learning, 16(3), 341-358.

Khalil, H., \& Ebner, M. (2014). MOOCs completion rates and possible methods to improve retention-A literature review. In J. Herrington, J. Viteli y M. Leikomaa (Eds.), EdMedia+ Innovate Learning (pp. 1236-1244). Chesapeake, VA: AACE.

Kim, T.-d., Yang, M.-y., Bae, J., Min, B.-a., Lee, I., \& Kim, J. (2017). Escape from infinite freedom: Effects of constraining user freedom on the prevention of dropout in an online learning context. Computers in Human Behavior, 66, 217-231.

Loken, E., Oravecz, Z., Tucker, C., \& Linder, F. J. (2015). Psychometric analysis of residence and MOOC assessments. In E. L. Usher, N. A. Mamaril, C. Li, D. R. Economy y M. S. Kennedy (Eds.), 122nd ASEE Annual Conference and Exposition: Making Value for Society (pp. 2612841261284114): American Society for Engineering Education.

Lortie-Forgues, H., Tian, J., \& Siegler, R. S. (2015). Why is learning fraction and decimal arithmetic so difficult? Developmental Review, 38, 201-221.

Misra, P. (2018). MOOCs for Teacher Professional Development: Reflections and Suggested Actions. Open Praxis, 10(1), 67-77.

Moreno-Marcos, P. M., Muñoz-Merino, P. J., Maldonado-Mahauad, J., Pérez-Sanagustín, M., Alario-Hoyos, C. \& Delgado Kloos, C. (2020). Temporal analysis for dropout prediction using self-regulated learning strategies in self-paced MOOCs. Computers \& Education, 145, 1-15.

Mourdi, Y., Sadgal, M., El Kabtane, H., \& Berrada Fathi, W. (2019). A machine learning-based methodology to predict learners' dropout, success or failure in MOOCs. International Journal of Web Information Systems, 15(5), 489-509. 
Normandi Atiaja Atiaja, L., \& Segundo Guerrero Proenza, R. (2016). MOOCs: Origin, characterization, principal problems and challenges in higher education. Journal of E-Learning and Knowledge Society, 12(1), 65-76.

Özgür, A., \& Yurdugül, H. (2015). The investigation of learner-assessment interaction in learning management systems. Master's thesis not published, Hacettepe University, Ankara.

Perez-Parras, J., \& Gomez-Galan, J. (2015). Knowledge and Influence of MOOC Courses on Initial Teacher Training. International Journal of Educational Excellence, 1(2), 81-99.

Perna, L. W., Ruby, A., Boruch, R. F., Wang, N., Scull, J., Ahmad, S., et al. (2014). Moving through MOOCs: Understanding the progression of users in massive open online courses. Educational Researcher, 43(9), 421-432.

Pilli, O., Admiraal, W., \& Salli, A. (2018). Moocs: Innovation or stagnation? Turkish Online Journal of Distance Education, 19(3), 169-181.

Ruiz, A. (2013). La reforma de la Educación Matemática en Costa Rica. Perspectiva de la praxis. Cuadernos de investigación y formación en educación matemática, 8(Especial), 7-111.

Salinas, P., Quintero, E. \& Sanchez, X. (2015). Math and motion: A (Coursera) MOOC to rethink math assessment. Lecture Notes in Computer Science, 9192, 313-324.

Samuelsen, J., \& Khalil, M. (2020). Study Effort and Student Success: A MOOC Case Study. In T. Tsiatsos \& M. E. Auer (Eds.), Proceedings of the 21th International Conference on Interactive Collaborative Learning (Vol. 1, pp. 215-226). Kos Island, Greece: Springer Verlag.

Segovia, I., \& Rico, L. (2011). Matemáticas para maestros de educación primaria. Madrid: Pirámide.

Thompson, N. (2019). What is classical item difficulty (P value)? Downloaded on 14/9/2019, from https://assess.com/2017/11/06/classical-item-difficulty-p-value/

Tømte, C. E. (2019). MOOCs in teacher education: institutional and pedagogical change? European Journal of Teacher Education, 42(1), 65-81.

Wambugu, P. W. (2018). Massive open online courses (MOOCs) for professional teacher and teacher educator development: A case of TESSA MOOC in Kenya. Universal Journal of Educational Research, 6(6), 1153-1157.

Wang, X., Hall, A. H., \& Wang, Q. (2019). Investigating the implementation of accredited massive online open courses (MOOCs) in higher education: The boon and the bane. Australasian Journal of Educational Technology, 35(3), 1-14.

Wen, Y., Tian, Y., Wen, B., Zhou, Q., Cai, G. \& Liu, S. (2020). Consideration of the local correlation of learning behaviors to predict dropouts from MOOCs. Tsinghua Science and Technology, 25(3), 336-347. 
Xiao, C., Qiu, H., \& Cheng, S. M. (2019). Challenges and opportunities for effective assessments within a quality assurance framework for MOOCs. Journal of Hospitality, Leisure, Sport and Tourism Education, 24, 1-16.

Xie, Z. (2020). Modelling the dropout patterns of MOOC learners. Tsinghua Science and Technology, 25(3), 313-324.

Yousef, A. M. F., Chatti, M. A., Schroeder, U., \& Wosnitza, M. (2014). What drives a successful MOOC? An empirical examination of criteria to assure design quality of MOOCs. In R. Huang, Kinshuk, D. G. Sampson, M. J. Spector \& N. S. Chen (Eds.), IEEE 14th International Conference on Advanced Learning Technologies (pp. 44-48). Athens, Greece: Institute of Electrical and Electronics Engineers Inc.

Youssef, M., Mohammed, S., Hamada, E. K., \& Wafaa, B. F. (2019). A predictive approach based on efficient feature selection and learning algorithms' competition: Case of learners' dropout in MOOCs. Education and Information Technologies, 24(6), 3591-3618.

Yuan, Q., Gao, Q., \& Chen, Y. (2017). A preliminary study on the learning assessment in massive open online courses. Lecture Notes in Computer Science, 10281, 592-602. 